Pacific Journal of Mathematics

REPRESENTATIONS ASSOCIATED WITH ELLIPTIC Walter RAymond ParRY 


\title{
REPRESENTATIONS ASSOCIATED WITH ELLIPTIC SURFACES
}

\author{
DAvid A. CoX AND WAlter R. PARRY
}

\begin{abstract}
An elliptic surface (over C) $f: X \rightarrow S$ with a section has two representations naturally associated to it: the first, the monodromy representation, is determined by the topology of $f$, while the second, the Galois representation, is determined by the arithmetic of the general fiber of $f$. The purpose of this paper is to study and compare the properties of these representations.
\end{abstract}

We will always assume that $f: X \rightarrow S$ is relatively minimal and that the $j$-invariant is nonconstant. We let $K$ denote the function field of $S$ and $E$ the general fiber of $f$. Then $E / K$ is an elliptic curve with $f: X \rightarrow S$ as its Néron model.

The Galois representation given by the action of $\operatorname{Gal}(\bar{K} / K)$ on the torsion points of $E(\bar{K})$ is studied first. Since $\mathbf{C}$ contains all roots of unity, this representation can be regarded as a continuous homomorphism

$$
\rho_{E / K}: \operatorname{Gal}(\bar{K} / K) \rightarrow \operatorname{SL}(2, \hat{\mathbf{Z}})=\prod_{p \text { prime }} \operatorname{SL}\left(2, \mathbf{Z}_{p}\right) .
$$

With the above hypothesis on $E / K$, it is known that the image of $\rho_{E / K}$, denoted $\operatorname{Im}\left(\rho_{E / K}\right)$, is open in $\operatorname{SL}(2, \hat{\mathbf{Z}})$ (see [5]). This naturally leads to the notion of level of $E / K$. In $\S 1$ we introduce this and study its basic properties. Then, in $\$ 2$, we show how to bound the level in terms of the behavior of the $j$-invariant and also in terms of the genus $g$ of $K$.

The monodromy representation (also called the homological invariant) of $f: X \rightarrow S$ is studied in $\S 3$. If $S_{0}=\{s \in S: f$ is smooth above $s\}$ and $X_{t}$ is the fiber over $t \in S_{0}$, then $\pi_{1}\left(S_{0}, t\right)$ acts on $H^{1}\left(X_{t}, \mathbf{Z}\right)$, giving us the monodromy representation

$$
\rho_{X / S}: \pi_{1}\left(S_{0}, t\right) \rightarrow \operatorname{SL}(2, \mathbf{Z}) .
$$

(The image is in $\operatorname{SL}(2, \mathbf{Z})$ because of Poincare duality.) We will show that the monodromy determines the Galois representation and that in some respects the monodromy is the more subtle invariant.

1. We will work in a slightly more general context than that of the introduction. Here, $K$ will be a field of characteristic zero containing all roots of unity, and $E / K$ will be an elliptic curve such that $\operatorname{Im}\left(\rho_{E / K}\right)$ is 
open in $\operatorname{SL}(2, \hat{\mathbf{Z}})$. This means that for some integer $n \geq 1$,

$$
\hat{\Gamma}(n) \subseteq \operatorname{Im}\left(\rho_{E / K}\right),
$$

where

$$
\hat{\Gamma}(n)=\{\gamma \in \operatorname{SL}(2, \hat{\mathbf{Z}}): \gamma \equiv 1 \bmod n\} .
$$

The level of $E / K$ is the smallest integer $n$ for which (1.1) holds. It can be shown that the level is actually the greatest common divisor of all such integers.

The level influences many things associated with $E / K$, as the next proposition shows.

Proposition 1.1. Let $E / K$ have level $n$.

(i) $\operatorname{End}_{K}(E)=\operatorname{End}_{\bar{K}}(\mathrm{E})=\mathbf{Z}$.

(ii) Let $\lambda: E \rightarrow E^{\prime}$ be a K-isogeny.

(a) If $\lambda$ is cyclic, then $\operatorname{deg}(\lambda) \mid n$.

(b) $E^{\prime} /$ K has level $n^{\prime}$, where $n^{\prime} \mid \operatorname{deg}(\lambda) n$. Thus $n^{\prime} \mid n^{2}$.

(iii) $E(K)_{\text {tor }}$ is $n$-torsion.

(iv) Let $p$ be prime and let

$$
\rho_{E / K, p}: \operatorname{Gal}(\bar{K} / K) \rightarrow \operatorname{SL}\left(2, \mathbf{F}_{p}\right)
$$

be the Galois representation on p-torsion points. If $p \nmid n$, then $\rho_{E / K, p}$ is surjective, and, if $p>5$, the converse is true.

Proof. Let $T(E)=\lim _{\leftarrow} E_{m}$, where $E_{m}=\{x \in E(\bar{K}): m x=0\}$. Then $T(E)=\Pi_{p} T_{p}(E) \cong \hat{\mathbf{Z}}^{2}$, where $T_{p}(E)$ is the usual Tate module over $\mathbf{Z}_{p}$. Every $K$-isogeny $\lambda: E \rightarrow E^{\prime}$ induces a map $T(E) \rightarrow T\left(E^{\prime}\right)$ which is represented by a matrix $A \in M(2, \hat{\mathbf{z}})$ such that $\operatorname{det}(A)=\operatorname{deg}(\lambda)$ for some choice of bases. Also, if a positive integer $k$ divides the entries of $A$, then $E_{k} \subseteq \operatorname{Ker}(\lambda)$. Since $\lambda$ is a $K$-isogeny,

$$
A \cdot \rho_{E / K}(\sigma)=\rho_{E^{\prime} / K}(\sigma) \cdot A
$$

for every $\sigma \in \operatorname{Gal}(\bar{K} / K)$.

To prove (i), take $\lambda \in \operatorname{End}_{K}(E)$. Since $\hat{\Gamma}(n) \subseteq \operatorname{Im}\left(\rho_{E / K}\right)$, (1.2) implies that $A$ centralizes $\hat{\Gamma}(n)$. Thus, $A$ is a homothety, which easily implies that $\operatorname{End}_{K}(E)=\mathbf{Z}$. Since this is true for all finite extensions of $K$, $\operatorname{End}_{\bar{K}}(E)=\mathbf{Z}$.

We now prove (ii). Since two isogenous elliptic curves are isogenous via a cyclic isogeny, $\lambda$ may be taken to be cyclic. This implies that bases of 
$T(E)$ and $T\left(E^{\prime}\right)$ can be chosen such that $A=\left(\begin{array}{ll}1 & 0 \\ 0 & N\end{array}\right)$, where $N=\operatorname{deg}(\lambda)$. Since $\hat{\Gamma}(n) \subseteq \operatorname{Im}\left(\rho_{E / K}\right),(1.2)$ implies

$$
A \hat{\Gamma}(n) A^{-1} \subseteq \operatorname{Im}\left(\rho_{E^{\prime} / K}\right) .
$$

Thus $N \mid n$ because $A \hat{\Gamma}(n) A^{-1} \subseteq \operatorname{SL}(2, \hat{\mathbf{z}})$, and $n^{\prime} \mid N n$ because $\hat{\Gamma}(N n) \subseteq$ $A \hat{\Gamma}(n) A^{-1} \subseteq \operatorname{Im}\left(\rho_{E^{\prime} / K}\right)$.

Now (iii) is clear because any element of $E(K)_{\text {tor }}$ defines a cyclic $K$-isogeny whose degree is the order of the element.

To prove (iv), note that $\hat{\Gamma}(n)=\Pi_{p} \Gamma\left(p^{v_{p}(n)}\right)_{p}$, where $\Gamma\left(p^{r}\right)_{p}=\{\gamma \in$ $\left.\operatorname{SL}\left(2, \mathbf{Z}_{p}\right): \gamma \equiv 1 \bmod p^{r}\right\}$. Thus the natural map

$$
\hat{\Gamma}(n) \rightarrow \operatorname{SL}\left(2, \mathbf{F}_{p}\right)
$$

is surjective when $p \nmid n$. The converse follows easily from [10, IV.3, Lemma 5].

If $E / K$ has finite level and $L$ is a finite extension of $K$, then $E / L$ clearly also has finite level. It is possible to estimate how much the level can change as follows.

Proposition 1.2. Let $E / K$ have level $n$, and let $L$ be a finite extension of $K$. Then $E / L$ has level $n^{\prime}$, where $n^{\prime} \leq[L: K] n$.

Proof. Let $G=\operatorname{Im}\left(\rho_{E / L}\right) \cap \hat{\Gamma}(n)$. Since $\hat{\Gamma}(n) \subseteq \operatorname{Im}\left(\rho_{E / K}\right)$, it follows that $[\hat{\Gamma}(n): G]$ divides $\left[\operatorname{Im}\left(\rho_{E / K}\right): \operatorname{Im}\left(\rho_{E / L}\right)\right]=[L: K]$. However:

The map $G \rightarrow G \cap \operatorname{SL}(2, Z)$ gives a bijection between open subgroups of $\operatorname{SL}(2, \hat{\mathbf{Z}})$ and congruence subgroups of $\operatorname{SL}(2, \mathbf{Z})$. This bijection preserves level, index, normal subgroups and quotients.

Let $\Gamma=G \cap \operatorname{SL}(2, \mathbf{Z})$. Then $\Gamma \subseteq \Gamma(n)$ and $\Gamma$ has level $n^{\prime}$, hence it suffices to prove that

$$
n^{\prime} \leq[\Gamma(n): \Gamma] n \text {. }
$$

When $n=1,(1.4)$ is proved in [2, Theorem 4.2], and the proof easily generalizes to the case when $n>1$.

Sometimes $E / L$ has finite level even when $L$ is an infinite extension of $K$. The most interesting example is when $L=K_{\mathrm{ab}}$, the maximal Abelian extension of $K$. In this case, Serre noticed (see [11, Remark, p. $300])$ that $E / K_{\mathrm{ab}}$ has finite level. We can estimate the level of $E / K_{\mathrm{ab}}$ as follows. 
TheOREM 1.3. Let $E / K$ have level $n$. Then $E / K_{\mathrm{ab}}$ has level $n^{\prime}$, where $n^{\prime} \mid 12 n^{2}$.

Proof. By Serre's result, $\operatorname{Im}\left(\rho_{E / K_{\mathrm{ab}}}\right)$ is a normal subgroup of $\operatorname{Im}\left(\rho_{E / K}\right)$ of finite index and Abelian qotient. Since $\hat{\Gamma}(n) \subseteq \operatorname{Im}\left(\rho_{E / K}\right)$, we see that $G=\operatorname{Im}\left(\rho_{E / K_{\mathrm{ab}}}\right) \cap \hat{\Gamma}(n)$ is normal in $\hat{\Gamma}(n)$, again with finite index and Abelian quotient. It suffices to prove that $\hat{\Gamma}\left(12 n^{2}\right) \subseteq G$.

We may assume that $G$ is the closure of the commutator subgroup of $\hat{\Gamma}(n)$. Using the notation of the proof of Proposition 1.1(iv), we have $\hat{\Gamma}(n)=\Pi_{p} \Gamma\left(p^{v_{p}(n)}\right)_{p}$. Then $G$ is also a product: $G=\Pi_{p} G_{p}$.

Let $H$ be the closure of the commutator subgroup of $\operatorname{SL}(2, \hat{\mathbf{Z}})$. One easily sees that $H=\Pi_{p} H_{p}$, where

\section{(1.5) $H_{p}=\operatorname{SL}\left(2, \mathbf{Z}_{p}\right)$ for $p>3$;}

$\left(\begin{array}{ll}2 & 1 \\ 1 & 1\end{array}\right) ;$ and

(1.6) $H_{3}$ has index 3 in $\operatorname{SL}\left(2, \mathbf{Z}_{3}\right)$ and is generated by $\Gamma(3)_{3},\left(\begin{array}{rr}0 & -1 \\ 1 & 0\end{array}\right)$ and

(1.7) $H_{2}$ has index 4 in $\operatorname{SL}\left(2, \mathbf{Z}_{2}\right)$ and is generated by $\Gamma(4)_{2},\left(\begin{array}{ll}0-1 \\ 0-1\end{array}\right)$ and $\left(\begin{array}{rr}-1 & 2 \\ 0 & -1\end{array}\right)$.

Fix a prime $p$ and let $r=v_{p}(n)$. Then $G_{p}$ is the closure of the commutator subgroup of $\Gamma\left(p^{r}\right)_{p}$. We will show that

(1.8) $G_{p}= \begin{cases}H_{p} \cap \Gamma\left(p^{2 r}\right)_{p} & \text { if } p \neq 2 \text { or } r=0 \\ \Gamma\left(2^{2 r}\right)_{2} \cap \Gamma_{0}\left(2^{2 r+1}\right)_{2} \cap \Gamma_{0}\left(2^{2 r+1}\right)_{2}^{t} & \text { if } p=2 \text { and } r>0\end{cases}$

where the subscript " 0 " has the usual meaning and the superscript " $t$ " means transpose. The theorem follows immediately from (1.8), and by computing indices, one also obtains the inequality

$$
\left[\hat{\Gamma}(n): \operatorname{Im}\left(\rho_{E / K_{\mathrm{ab}}}\right) \cap \hat{\Gamma}(n)\right] \leq 12 n^{3} .
$$

This will be useful later.

Before proving (1.8), note that it is closely related to a result of Lang and Trotter which describes the closure of the commutator subgroup of $\left\{\gamma \in \mathrm{GL}\left(2, \mathbf{Z}_{p}\right): \gamma \equiv 1 \bmod p^{r}\right\}($ see $[8$, p. 95 and pp. 163-173]). The only difference occurs when $p=2$.

Let $\tilde{G}_{p}$ denote the right hand side of (1.8). The case $r=0$ is trivial. To handle the case $r>0$, we start with the following three simple observations.

(1.10) The commutators of $\operatorname{sl}\left(2, \mathbf{Z}_{p}\right)$ generate the subgroup

$$
\Lambda=\left\{\left(\begin{array}{ll}
a & b \\
c & d
\end{array}\right) \in \operatorname{sl}\left(2, \mathbf{Z}_{p}\right): b \equiv c \equiv 0 \bmod 2\right\} .
$$


(1.11) If $1+p^{r} A$ is in $\Gamma\left(p^{r}\right)_{p}$, then $\operatorname{tr}(A) \equiv 0 \bmod p^{r}$.

(1.12) If $x=1+p^{r} A$ and $y=1+p^{r} B$ are in $\Gamma\left(p^{r}\right)_{p}$, then

$$
\begin{aligned}
x y x^{-1} y^{-1}= & 1+p^{2 r}[A, B] \\
& +p^{3 r}[A, B]\left(\sum_{k=1}^{\infty}(-1)^{k} p^{(k-1) r}\left(\sum_{i+j=k} A^{i} B^{j}\right)\right) .
\end{aligned}
$$

These facts immediately imply that $G_{p} \subseteq \tilde{G}_{p}$. For the opposite inclusion, we will show that if $1+p^{k r} A$ is in $\tilde{G}_{p}, k \geq 2$, then there are $x_{l}, y_{i} \in \Gamma\left(p^{r}\right)_{p}$, $1 \leq i \leq 3$, such that

$$
1+p^{k r} A=\prod_{i=1}^{3} x_{i} y_{i} x_{i}^{-1} y_{i}^{-1} \quad \bmod p^{(k+1) r} .
$$

This implies that $\tilde{G}_{p}$ consists of convergent infinite products of commutators of elements of $\Gamma\left(p^{r}\right)_{p}$, proving (1.8).

To show that (1.13) holds, first note that $p^{(k-2) r} A \equiv \tilde{A} \bmod p^{(k-1) r}$ for some $\tilde{A} \in \Lambda$. By (1.10), $\tilde{A}=\sum_{i=1}^{3}\left[A_{i}, B_{i}\right]$, where $A_{i}$ and $B_{i}$ are nilpotent and $\left[A_{i}, B_{i}\right] \equiv 0 \bmod p^{(k-2) r}$. Then $x_{i}=1+p^{r} A_{i}$ and $y_{i}=1+p^{r} B_{i}$ lie in $\Gamma\left(p^{r}\right)_{p}$, and (1.13) follows from (1.12).

Since $E / K_{\mathrm{ab}}$ has finite level, it follows that $E\left(K_{\mathrm{ab}}\right)_{\mathrm{tor}}$ is finite. This fact was noticed by Mazur in [9, Proposition 6.12]. Combining Theorem 1.3 and Proposition 1.1(iii), we get the following more explicit result.

COROLlaRY 1.4. If $E / K$ has level $n$, then $E\left(K_{\mathrm{ab}}\right)_{\text {tor }}$ is $12 n^{2}$-torsion.

We next cast our results in field theoretic terms. Let $K_{\text {tor }}$ be the field obtained from $K$ by adjoining the coordinates of points in $E(\bar{K})_{\text {tor }}$.

COROLlaRY 1.5. If $E / K$ has level $n$, then

$$
\left[K_{\mathrm{ab}} \cap K_{\mathrm{tor}}: K\right] \leq 12 n^{5} \prod_{\left.p\right|^{n}}\left(1-p^{-2}\right) .
$$

Proof. Let $L=K_{\mathrm{ab}} \cap \mathrm{K}_{\text {tor }}$. Then $[L: K]=\left[\operatorname{Gal}\left(K_{\text {tor }} / K\right)\right.$ : $\left.\operatorname{Gal}\left(K_{\text {tor }} / L\right)\right]$. It is well-known that $\operatorname{Gal}\left(K_{\text {tor }} / K\right) \cong \operatorname{Im}\left(\rho_{E / K}\right)$ and $\operatorname{Gal}\left(K_{\text {tor }} / L\right) \cong \operatorname{Im}\left(\rho_{E / K_{\mathrm{ab}}}\right)$. Thus $[L: K]=\left[\operatorname{Im}\left(\rho_{E / K}\right): \operatorname{Im}\left(\rho_{E / K_{\mathrm{ab}}}\right)\right]$. Since $\hat{\Gamma}(n) \subseteq \operatorname{Im}\left(\rho_{E / K}\right)$, we get

$$
[L: K] \leq\left[\operatorname{Im}\left(\rho_{E / K}\right): \hat{\Gamma}(n)\right]\left[\hat{\Gamma}(n): \operatorname{Im}\left(\rho_{E / K_{\mathrm{ab}}}\right) \cap \hat{\Gamma}(n)\right],
$$


and then (1.9) implies

$$
[L: K] \leq\left[\operatorname{Im}\left(\rho_{E / K}\right): \hat{\Gamma}(n)\right] \cdot\left(12 n^{3}\right) .
$$

But $\operatorname{Im}\left(\rho_{E / K}\right) \subseteq \operatorname{SL}(2, \hat{\mathbf{Z}})=\hat{\Gamma}(1)$, so that by (1.3) and (1.4) we have

$$
n \leq\left[\operatorname{SL}(2, \hat{\mathbf{Z}}): \operatorname{Im}\left(\rho_{E / K}\right)\right] \text {. }
$$

The index of $\hat{\Gamma}(n)$ in $\operatorname{SL}(2, \hat{\mathbf{Z}})$ is known, yielding

$$
\left[\operatorname{Im}\left(\rho_{E / K}\right): \hat{\Gamma}(n)\right] \leq n^{2} \prod_{\left.p\right|^{n}}\left(1-p^{-2}\right) .
$$

This formula and (1.14) give the desired estimate for [ $L: K]$.

Besides the level, there are other invariants of $\operatorname{Im}\left(\rho_{E / K}\right)$. One of the most natural is the index of $\operatorname{Im}\left(\rho_{E / K}\right)$ in $\operatorname{SL}(2, \hat{\mathbf{Z}})$. We have the following relation between level and index.

PROPOSITION 1.6.

(i) If $E / K$ has level $n$, then

$$
n \leq\left[\operatorname{SL}(2, \hat{\mathbf{z}}): \operatorname{Im}\left(\rho_{E / K}\right)\right] \leq n^{3} \prod_{\left.p\right|^{n}}\left(1-p^{-2}\right) .
$$

(ii) The index $\left[\operatorname{SL}(2, \hat{\mathbf{Z}}): \operatorname{Im}\left(\rho_{E / K}\right)\right]$ is an isogeny invariant of $E / K$; the level is not.

Proof. The proof of Corollary 1.5 gives (i). To prove (ii), suppose that $E$ and $E^{\prime}$ are $K$-isogenous. By (1.3), $\Gamma=\operatorname{Im}\left(\rho_{E / K}\right) \cap \operatorname{SL}(2, \mathbf{Z})$ and $\Gamma^{\prime}=$ $\operatorname{Im}\left(\rho_{E^{\prime} / K}\right) \cap \operatorname{SL}(2, \mathbf{Z})$ are congruence subgroups, and we need only show that they have the same index in $\operatorname{SL}(2, Z)$. From (1.2) it follows that $\Gamma$ and $\Gamma^{\prime}$ are conjugate in $\operatorname{SL}(2, \mathbf{R})$. Thus their fundamental domains have the same volume, therefore $\pm \Gamma$ and $\pm \Gamma^{\prime}$ have the same index in $\operatorname{SL}(2, \mathbf{Z})$ and thus $\Gamma$ and $\Gamma^{\prime}$ have the same index in $\operatorname{SL}(2, \mathbf{Z})$. In $\S 3$, we will give examples to show that the level is not an isogeny invariant.

While we are principally concerned with elliptic curves over function fields, we now comment on the arithmetic case. An elliptic curve $E$ over a number field $K$ has a Galois representation

$$
\rho_{E / K}: \operatorname{Gal}(\bar{K} / K) \rightarrow \mathrm{GL}(2, \hat{\mathbf{Z}}),
$$

and Serre has proved that $\operatorname{Im}\left(\rho_{E / K}\right) \cong \operatorname{GL}(2, \hat{\mathbf{Z}})$ has finite index when $E$ has no complex multiplication (see [11]). If $K_{\text {cyc }}$ is $K$ with all roots of unity 
adjoined, it follows that $E / K_{\text {cyc }}$ has finite level, which we may define to be the level of $E / K$. The results of this section then provide useful information about the arithmetic of $E / K$. (Lang and Trotter have defined an invariant of $\operatorname{Im}\left(\rho_{E / K}\right) \subseteq \mathrm{GL}(2, \hat{\mathbf{z}})$ analogous to the level: in the language of $[8$, p. 18], one takes the smallest integer which is stable and splitting for $G=\operatorname{Im}\left(\rho_{E / K}\right)$.)

2. In this section we return to the situation of the introduction, where $E$ is an elliptic curve over a function field $K$ in one variable over $\mathbf{C}$, and the $j$-invariant is nonconstant. The Néron model of $E / K$ is an elliptic surface $f: X \rightarrow S$. Our goal here is to get effectively computable bounds for the level of $E / K$.

We first show how the $j$-invariant influences the level.

Proposition 2.1. Let $E / K$ have level $n$. Then

(i) $n \leq 2 \operatorname{deg}(j)$,

(ii) $n \mid 2 \mathrm{LCM}\{b: j$ has a pole of order $b\}$.

Proof. Let $H$ be the image of $\operatorname{Im}\left(\rho_{E / K}\right)$ in $\operatorname{SL}(2, \mathbf{Z} / n \mathbf{Z})$. Then $E / K$ has a level $H$-structure in the sense of $[3, \S 3.1]$. Since $\Gamma=\operatorname{Im}\left(\rho_{E / K}\right) \cap$ $\operatorname{SL}(2, \mathbf{Z})$ is the inverse image of $H$ in $\operatorname{SL}(2, \mathbf{Z}),[3, \S 5]$ gives us a commutative diagram

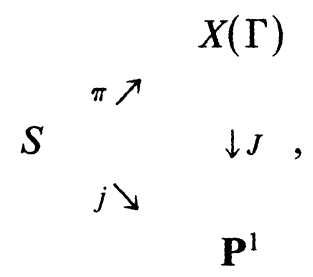

where $X(\Gamma)=\Gamma \backslash \mathfrak{S}^{*}$, and $J$ is the natural map induced by $\Gamma \subseteq \operatorname{SL}(2, \mathbf{Z})$.

From (2.1), we see that $\operatorname{deg}(J) \mid \operatorname{deg}(j)$. Since $\operatorname{deg}(J)=[\operatorname{SL}(2, \mathbf{Z})$ : $\pm \Gamma]$, it follows from (1.4) that

$$
m \leq[\operatorname{SL}(2, \mathbf{Z}): \pm \Gamma] \leq \operatorname{deg}(j),
$$

where $m$ is the level of $\pm \Gamma$.

By [15, Theorem 2], we have

$$
\begin{aligned}
m & =\operatorname{LCM}\{\text { widths of cusps of } \pm \Gamma\} \\
& =\operatorname{LCM}\{b: J \text { has a pole of order } b\} .
\end{aligned}
$$

Then (2.1) implies that $m \mid \operatorname{LCM}\{b: j$ has a pole of order $b\}$. 
It remains to relate $m$, the level of $\pm \Gamma$, to $n$, the level of $\Gamma$. Since $[ \pm \Gamma: \Gamma] \leq 2$, it follows that $[\Gamma(m): \Gamma \cap \Gamma(m)] \leq 2$, and since $\Gamma \cap \Gamma(m)$ has level $n,(1.4)$ gives that

$$
n \leq[\Gamma(m): \Gamma \cap \Gamma(m)] \cdot m \leq 2 m .
$$

Hence $n=m$ or $n=2 m$, and the proposition follows.

In $§ 3$, we will give examples to show that the factor of 2 is necessary in both parts of Proposition 2.1.

A more striking result is that the level of $E / K$ is bounded by a constant depending only on the genus of the base field $K$. Recall that $K$ is the function field of the Riemann surface $S$.

THEOREM 2.2. Let $E / K$ have level $n$, and let $S$ have genus $g$.

(i) If $g=0$, then $n=O(1)$.

(ii) If $g \geq 1$, then $n=24 g+O\left(g^{1 / 2}\right)$.

(iii) If $p$ is a prime dividing $n$, then $p \leq 12 g+13$.

Proof. By (1.3), $\Gamma=\operatorname{Im}\left(\rho_{E / K}\right) \cap \operatorname{SL}(2, \mathbf{Z})$ is a congruence subgroup of level $n$. Since $j$ is nonconstant, the map $\pi: S \rightarrow X(\Gamma)$ of $(2.1)$ is surjective. Thus, letting $\bar{g}$ denote the genus of $X(\Gamma)$, we have

$$
\bar{g} \leq g .
$$

Let $\bar{\Gamma}$ be the image of $\Gamma$ in $\operatorname{PSL}(2, Z)$, and let its level be $\bar{n}$. Then $\bar{g}$ is also the genus of $X(\bar{\Gamma})$, and we can use the following results of [2] to relate $\bar{g}$ and $\bar{n}$.

THEOREM 2.3. Let $\bar{\Gamma} \subseteq \operatorname{PSL}(2, \mathbf{Z})$ be a congruence subgroup of level $\bar{n}$, and let $\bar{g}$ be the genus of $X(\bar{\Gamma})$.

(i) If $\bar{g}=0$, then $\bar{n}=O(1)$.

(ii) If $\bar{g} \geq 1$, then $\bar{n}=12 \bar{g}+O\left(\bar{g}^{1 / 2}\right)$.

(iii) If $p$ is a prime dividing $\bar{n}$, then $p \leq 12 \bar{g}+13$.

Proof. See Corollary 4.7 (when $\bar{g}=0$ ), Corollary 4.8 and Proposition 4.9 in [2].

Since $\bar{n}$ is also the level of $\pm \Gamma, \bar{n}=n$ or $\bar{n}=n / 2$, and the theorem follows immediately from (2.2) and Theorem 2.3.

More precise versions of (i) and (ii) in Theorem 2.2 may be stated as follows.

(i)' If $g=0$, then $n \leq 64$. 
(ii)' If $g \geq 1$, then

$$
n \leq 24 g+13(48 g+121)^{1 / 2}+145 \text {. }
$$

These statements follow from a more precise version of Theorem 2.3 which appears in the preprint version of [2]. (Specifically, see Corollary 4.11 and Table 5.1 in the preprint, and note that the group of level 36, resp. 48, in $\operatorname{PSL}(2, \mathbf{Z})$ in Table 5.1 is not the image of a group of level 72, resp. 96 , in $\operatorname{SL}(2, \mathbf{Z})$.)

Here is a corollary of Theorem 2.2(iii) and Proposition 1.1(iv).

COROLlARY 2.4. With the above notation, the Galois representation on p-torsion points

$$
\rho_{E / K, p}: \operatorname{Gal}(\bar{K} / K) \rightarrow \operatorname{SL}\left(2, \mathbf{F}_{p}\right)
$$

is surjective for all primes $p>12 \mathrm{~g}+13$.

Another corollary of Theorem 2.2 is the following finiteness result.

COROLlaRY 2.5. For a fixed function field $K$ over $\mathbf{C}$, there are only finitely many possibilities for the image of the Galois representation $\rho_{E / K}$.

Since there are only finitely many congruence subgroups $\Gamma$ of $\operatorname{SL}(2, \mathbf{Z})$ such that $X(\Gamma)$ has a given genus (proved by Thompson in [14]), this corollary was already known.

Given the strength of these theorems, one might hope for similar results in the number field case. Here, recall that $E$ is an elliptic curve without complex multiplication over a number field $K$. Little is known about the size of $\operatorname{Im}\left(\rho_{E / K}\right) \subseteq \mathrm{GL}(2, \hat{\mathbf{Z}})$, although some examples have been computed (see [8] and [11]). In analogy with Proposition 2.1, Serre (see [11, §5]) has shown, when $K=\mathbf{Q}$, how to bound the primes dividing the level in terms of the reduction data of $E / \mathbf{Q}$. It should be possible to bound the level itself using the reduction data. The analog of Theorem 2.2 is quite a different matter. Given the present state of knowledge, one cannot even reasonably conjecture such a result. The number field case is much deeper than the function field case.

3. Let $E / K$ be as in $\S 2$, and let $f: X \rightarrow S$ be its Néron model. We now study the monodromy representation

$$
\rho_{X / S}: \pi_{1}\left(S_{0}, t\right) \rightarrow \mathrm{SL}(2, \mathbf{Z})
$$


defined in the introduction. The image $\Gamma$ of $\rho_{X / S}$ in $\operatorname{SL}(2, \mathbf{Z})$ is called the global monodromy group of $f: X \rightarrow S$. Both $\rho_{X / S}$ and $\Gamma$ are topological invariants in the sense that they are uniquely determined up to $\operatorname{SL}(2, \mathbf{Z})$ conjugacy by the topology of $f: X \rightarrow S$ and the orientation induced on the smooth fibers of $f$. Stiller has studied the basic properties of $\Gamma$ :

Proposition 3.1. Let $\Gamma$ be the global monodromy group of $f: X \rightarrow S$.

(i) $\Gamma$ has finite index in $\mathrm{SL}(2, \mathbf{Z})$.

(ii) There is a commutative diagram

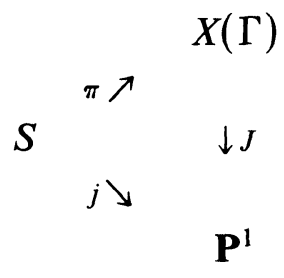

where $J$ is the natural map induced by $\Gamma \subseteq \mathrm{SL}(2, \mathbf{Z})$.

(iii) $[\operatorname{SL}(2, \mathbf{Z}): \pm \Gamma] \mid \operatorname{deg}(j)$.

(iv) $[\operatorname{SL}(2, \mathbf{Z}): \pm \Gamma]$ is an isogeny invariant of $E / K$.

Proof. See [13, §§1 and 2].

Stiller also shows that other interesting invariants of $E / K$ are isogeny invariants. Propositions 1.6(ii) and 2.1(i) were inspired by parts (iii) and (iv) of Proposition 3.1.

Results such as the above lead one to expect a close relation between the Galois and monodromy representations. To state the relation precisely, we need to recall some facts.

(3.1) There is a continuous homomorphism

$$
\left(\rho_{X / S}\right)^{\hat{\imath}}: \pi_{1}\left(S_{0}, t\right)^{\wedge} \rightarrow \operatorname{SL}(2, \hat{\mathbf{z}})
$$

(where ${ }^{\wedge}$ denotes profinite completion) such that the diagram

$$
\begin{array}{ccc}
\pi_{1}\left(S_{0}, t\right) & \stackrel{\rho_{X / S}}{\rightarrow} & \mathrm{SL}(2, \mathbf{Z}) \\
\cap \mid & & \cap \mid \\
\pi_{1}\left(S_{0}, t\right)^{\wedge} & \stackrel{\left(\rho_{X / S}\right)^{\hat{n}}}{\rightarrow} & \mathrm{SL}(2, \hat{\mathbf{Z}})
\end{array}
$$

commutes.

(3.2) $\pi_{1}\left(S_{0}, t\right)^{\wedge}$ is isomorphic to the étale fundamental group $\pi_{1}^{\mathrm{et}}\left(S_{0}, t\right)$.

(3.3) There is a continuous surjection

$$
g: \operatorname{Gal}(\bar{K} / K) \rightarrow \pi_{1}^{\mathrm{et}}\left(S_{0}, t\right) .
$$


Our basic result is that $\rho_{X / S}$ determines $\rho_{E / K}$ as follows.

THEOREM 3.2. The diagram

$$
\begin{array}{ccc}
\operatorname{Gal}(\bar{K} / K) & \stackrel{\rho_{E / K}}{\rightarrow} & \mathrm{Sl}(2, \hat{\mathbf{Z}}) \\
g \downarrow & & \uparrow\left(\rho_{X / S}\right)^{\hat{}} \\
\pi_{1}^{\mathrm{et}}\left(S_{0}, t\right) & \stackrel{\sim}{\rightarrow} & \pi_{1}\left(S_{0}, t\right)^{\hat{}}
\end{array}
$$

is commutative.

Proof. Let $X_{t}$ be the fiber of $f: X \rightarrow S$ over $t$, and let $E_{n}=\{x \in E(\bar{K})$ : $n x=0\}$. Then it suffices to find isomorphisms

$$
\phi_{n}: E_{n} \stackrel{\sim}{\rightarrow} H^{1}\left(X_{t}, \mathbf{Z} / n \mathbf{Z}\right),
$$

compatible with the natural inclusions $\mathbf{Z} / n \mathbf{Z} \subseteq \mathbf{Z} / m \mathbf{Z}$ and $E_{n} \subseteq E_{m}$ (when $n \mid m$ ), such that the diagrams

$$
\begin{array}{ccc}
\operatorname{Gal}(\bar{K} / K) & \stackrel{\rho_{1}}{\rightarrow} & \operatorname{Aut}\left(E_{n}\right) \\
\downarrow & & \downarrow 2 \operatorname{Aut}\left(\phi_{n}\right) \\
\pi_{1}\left(S_{0}, t\right) \wedge & \stackrel{\rho_{2}}{\rightarrow} & \operatorname{Aut}\left(H^{1}\left(X_{t}, \mathbf{Z} / n \mathbf{Z}\right)\right)
\end{array}
$$

commute for all $n$, where $\rho_{1}$ and $\rho_{2}$ are determined by $\rho_{E / K}$ and $\rho_{X / S}$ respectively.

The map sending 1 to $e^{2 \pi i / n}$ induces compatible isomorphisms $\mathbf{Z} / n \mathbf{Z}$ $\cong \boldsymbol{\mu}_{n}$. Thus, in (3.4), we can replace $\mathbf{Z} / n \mathbf{Z}$ by $\boldsymbol{\mu}_{n}$.

The map $\rho_{2}$, restricted to $\pi_{1}\left(S_{0}, t\right)$, describes the locally constant sheaf $R^{1} f_{*} \mu_{n}$ on $S_{0}$. Working in the étale topology, there is a locally constant sheaf $R_{\text {et }}^{1} f_{*} \mu_{n}$ which is described by a map

$$
\rho_{3}: \pi_{1}^{\mathrm{et}}\left(S_{0}, t\right) \rightarrow \operatorname{Aut}\left(H_{\mathrm{et}}^{\mathrm{l}}\left(X_{t}, \boldsymbol{\mu}_{n}\right)\right) .
$$

The comparison theorem of [1, XVI 4.1] gives us compatible commutative diagrams

$$
\begin{array}{ccc}
\pi_{1}^{\text {et }}\left(S_{0}, t\right) & \stackrel{\rho_{3}}{\rightarrow} & \operatorname{Aut}\left(H_{\mathrm{et}}^{1}\left(X_{t}, \mu_{n}\right)\right) \\
\downarrow l & & \downarrow l \\
\pi_{1}\left(S_{0}, t\right) \hat{\wedge} & \stackrel{\rho_{2}}{\rightarrow} & \operatorname{Aut}\left(H^{1}\left(X_{t}, \mu_{n}\right)\right) .
\end{array}
$$

Next, let the map $\xi: \operatorname{Spec}(\bar{K}) \rightarrow S_{0}$ be induced by the inclusion $K \subseteq \bar{K}$. Then the geometric point $t \in S_{0}$ gives us a specialization $\xi \rightarrow t$. 
The specialization morphisms

$$
\begin{aligned}
\pi_{1}^{\mathrm{et}}\left(S_{0}, t\right) & \rightarrow \pi_{1}^{\mathrm{et}}\left(S_{0}, \xi\right) \\
H_{\mathrm{et}}^{\mathrm{l}}\left(X_{\xi}, \mu_{n}\right) & \rightarrow H_{\mathrm{et}}^{\mathrm{l}}\left(X_{t}, \boldsymbol{\mu}_{n}\right)
\end{aligned}
$$

are isomorphisms by [1, XVI 2.2 and 2.3], and we can replace $t$ by $\xi$ in the bottom row of (3.5).

Finally, note that $\pi_{1}^{\text {et }}(\operatorname{Spec}(K), \xi) \cong \operatorname{Gal}(\bar{K} / K)$, and that the isomorphism

$$
H_{\mathrm{et}}^{1}\left(X_{\xi}, \mu_{n}\right) \cong E_{n}
$$

of [1, IX 4.7] is compatible with the Galois action (and also with the usual maps $\mu_{n} \subseteq \mu_{m}$ and $E_{n} \subseteq E_{m}$ ). This implies that $\rho_{1}$ can be identified in a natural way with $\rho_{3} \circ \delta$, where

$$
\delta: \pi_{1}^{\mathrm{et}}(\operatorname{Spec}(K), \xi) \rightarrow \pi_{1}^{\mathrm{et}}\left(S_{0}, \xi\right)
$$

is induced by the map $\operatorname{Spec}(K) \rightarrow S_{0}$. Then (3.5)-(3.7) give us the desired maps $\phi_{n}$, and the theorem follows.

This theorem also proves the well-known fact that the Galois representation is unramified over $S_{0}$ (i.e., where $E / K$ has good reduction).

Here are some simple corollaries of Theorem 3.2.

COROLlary 3.3. Given $E / K$, let $\Gamma$ be the global monodromy group of its Néron model.

(i) $\operatorname{Im}\left(\rho_{E / K}\right)$ is the closure of $\Gamma$ in $\operatorname{SL}(2, \hat{\mathbf{Z}})$.

(ii) $\operatorname{Im}\left(\rho_{E / K}\right) \cap \operatorname{SL}(2, \mathbf{Z})$ is the smallest congruence subgroup of $\operatorname{SL}(2, \mathbf{Z})$ containing $\Gamma$.

COROllary 3.4. $\operatorname{Im}\left(\rho_{E / K}\right)$ and the level of $E / K$ are topological invariants of the Neron model of $E / K$.

We can now give the example promised in Proposition 1.6(ii). In [13, §3], Stiller constructs isogenous elliptic curves $E$ and $\tilde{E}$ over $\mathbf{C}(t)$ such that their Néron models have global monodromy groups $\Gamma(2)$ and $\Gamma_{0}(4)$ respectively. It follows from Corollary 3.3 that $E / \mathbf{C}(t)$ has level 2, while $\tilde{E} / \mathbf{C}(t)$ has level 4 . Note that this is the maximum change of level allowed by Proposition 1.1(ii).

Since the global monodromy group $\Gamma$ determines $\operatorname{Im}\left(\rho_{E / K}\right)$, it is natural to ask if the converse is true. If $\Gamma$ were always a congruence subgroup of $\operatorname{SL}(2, \mathbf{Z})$, then the converse would follow immediately from Corollary 3.3. However, the following shows that $\Gamma$ can be any subgroup of $\operatorname{SL}(2, \mathbf{Z})$ of finite index. 
Proposition 3.5. Let $\Gamma$ be a subgroup of finite index in $\mathrm{SL}(2, \mathbf{Z})$. Then there is an elliptic curve $E / K$, where $K$ is the function field of $X(\Gamma)$, whose Néron model has $\Gamma$ as its global monodromy group.

Proof. Let $\bar{\Gamma}$ be the image of $\Gamma$ in $\operatorname{PSL}(2, \mathbf{Z})$, and let $\mathcal{E}$ be the set of elliptic points of $\operatorname{SL}(2, \mathbf{Z})$ acting on $\mathfrak{S}$. Then $\bar{\Gamma}$ acts freely on $\mathfrak{S}-\mathcal{E}$ with quotient, say, $S_{0}$, giving us a surjective homomorphism

$$
\bar{\rho}: \pi_{1}\left(S_{0}\right) \rightarrow \bar{\Gamma}
$$

Suppose there is a commutative diagram

$$
\begin{array}{ccc} 
& & \Gamma \\
\pi_{1}\left(S_{0}\right) & \rho \nearrow & \\
& & \downarrow \\
& \bar{\rho} \searrow & \\
& & \bar{\Gamma}
\end{array}
$$

Let $J: X(\bar{\Gamma}) \rightarrow \mathbf{P}^{1}$ be the natural map. Then $\rho$ belongs to $J$ in the sense of $[7, \S 8]$, so we can let $f: X \rightarrow X(\bar{\Gamma})$ be the basic member of $\mathscr{F}(\rho, J)$ (again, see $[7, \S 8])$. One easily checks that $\operatorname{Im}(\rho)$ is the global monodromy group. Thus, the generic fiber of $f$ will give the desired example, provided we can find a surjective map $\rho$ satisfying (3.8).

If $-1 \notin \Gamma$, then $\Gamma \rightarrow \bar{\Gamma}$ is an isomorphism, so that $\rho$ exists and is clearly surjective. (It is clear from $[12, \S 4]$ that this gives us the elliptic modular surface of $\Gamma$.)

Suppose that $-1 \in \Gamma$. Our above construction gives us a commutative diagram

$$
\begin{array}{ccc}
\pi_{1}\left(S_{0}\right) & \stackrel{\bar{\rho}}{\rightarrow} & \bar{\Gamma} \\
\cap \mid & & \cap \mid \\
\pi_{1}\left(\mathbf{P}^{1}-\{0,1, \infty\}\right) & \stackrel{\bar{\rho}_{1}}{\rightarrow} & \operatorname{PSL}(2, \mathbf{Z}) .
\end{array}
$$

where $\bar{\rho}_{1}$ is surjective. Since $\pi_{1}\left(\mathbf{P}^{1}-\{0,1, \infty\}\right)$ is free, $\bar{\rho}_{1}$ lifts to a homomorphism

$$
\rho_{1}: \pi_{1}\left(\mathbf{P}^{1}-\{0,1, \infty\}\right) \rightarrow \operatorname{SL}(2, \mathbf{Z})
$$

which is easily seen to be surjective. Then $\rho=\rho_{1 \mid \pi_{1}\left(S_{0}\right)}$ gives the desired surjective lift of $\bar{\rho}$.

We can now give the examples promised in the remarks following the proof of Proposition 2.1. Let $\Gamma$ be the commutator subgroup of $\operatorname{SL}(2, \mathbf{Z})$. 
Then (1.3) and (1.5)-(1.7) show that $-1 \notin \Gamma,[\operatorname{SL}(2, \mathbf{Z}): \Gamma]=12$ and, contrary to the claim of [12, Ex. 5.9], $\Gamma$ has level 12. The proof of Proposition 3.5 shows that the elliptic modular surface of $\Gamma$ has $\Gamma$ as its global monodromy group. Then the corresponding elliptic curve $E / K$ has level 12 by Corollary 3.3. The $j$-invariant of $E / K$ has only one pole, which is of order 6 (see [12, Ex. 5.9]), so that $6=\operatorname{deg}(j)=\operatorname{LCM}\{b: j$ has a pole of order $b$ \}. Thus, the factors of 2 in Proposition 2.1 are necessary.

A final question to ask is if the analog of Corollary 2.5 holds for the global monodromy group $\Gamma$ : for elliptic surfaces over a fixed Riemann surface $S$, are there only finitely many possibilities for $\Gamma$ ? The answer is no. To see this, note that by [6], there are infinitely many subgroups $\Gamma \subseteq \operatorname{SL}(2, \mathbf{Z})$ of finite index such that $X(\Gamma) \cong \mathbf{P}^{1}$. Given such a $\Gamma$, Proposition 3.5 gives us an elliptic surface $f: X \rightarrow \mathbf{P}^{1}$ with monodromy representation

$$
\rho_{X / \mathbf{P}^{1}}: \pi_{1}\left(S_{0}\right) \rightarrow \Gamma
$$

where $S_{0} \subseteq \mathbf{P}^{1}$ and $\rho_{X / \mathbf{P}^{1}}$ is surjective. If $S$ is any Riemann surface, we can find a map $\pi: S \rightarrow \mathbf{P}^{1}$ which is unramified above $\mathbf{P}^{1}-S_{0}$. Then the pullback of $f: X \rightarrow \mathbf{P}^{1}$ via $\pi$ gives us an elliptic surface over $S$ with $\Gamma$ as global monodromy group. This gives us infinitely many global monodromy groups $\Gamma$. Combining this with Corollary 2.5 , we get infinitely many elliptic surfaces over $S$ with distinct $\Gamma$ 's and the same $\operatorname{Im}\left(\rho_{E / K}\right)$. Thus, we see that the global monodromy group is a much more subtle invariant than the image of the Galois representation.

\section{REFERENCES}

[1] M. Artin, A. Grothendieck, and J. L. Verdier, Théorie des Topos et Cohomologie Étale des Schémas (SGA4), Lecture Notes in Math., vol. 305, Springer, New York, 1973.

[2] D. Cox and W. Parry, Genera of congruence subgroups in Q-quaternion algebras, J. Reine Angew. Math., to appear.

[3] P. Deligne and M. Rapoport, Les schémas de modules des courbes elliptiques, in Modular Functions of One Variable II, Lecture Notes in Math., vol. 349, Springer, New York, 1973.

[4] A. Grothendieck, Revêtements Étales et Groupe Fondemental (SGA1), Lecture Notes in Math., vol. 224, Springer, New York, 1971.

[5] J. Igusa, Fiber systems of Jacobian varieties III, Amer. J. Math., 81 (1959), 453-476.

[6] G. Jones, Triangular maps and non-congruence subgroups of the modular group, Bull. London Math. Soc., 11 (1979), 117-123.

[7] K. Kodaira, On compact analytic surfaces II, Annals of Math., 77 (1963), 563-626.

[8] S. Lang and H. Trotter, Frobenius Distributions in $\mathrm{GL}_{2}$-extensions, Lecture Notes in Math., vol. 504, Springer, New York, 1976.

[9] B. Mazur, Rational points of abelian varieties with values in towers of number fields, Inv. Math., 18 (1972), 183-266. 
[10] J.-P. Serre, Abelian l-adic Representations and Elliptic Curves, Benjamin, New York, 1968.

[11] Proprietes galoisiennes des points $d$ 'ordre fini des courbes elliptiques, Inv. Math., 15 (1972), 259-331.

[12] T. Shioda, On elliptic modular surfaces, J. Math. Soc. Japan, 24 (1972), 20-59.

[13] P. Stiller, Monodromy and invariants of elliptic surfaces, Pacific J. Math., 92 (1981), 433-452.

[14] J. Thompson, $A$ finiteness theorem for subgroups of $\operatorname{PSL}(2, \mathbf{R})$ which are commensurable with PSL $(2, \mathbf{Z})$, in The Santa Cruz conference on finite groups, Proceedings of Symposia in Pure Math., vol. 37, AMS, Providence, 1980.

[15] K. Wohlfahrt, An extension of F. Klein's level concept, Illinois J. Math., 8 (1964), 529-535.

Received September 3, 1982. First author's research supported by NSF Grant MCS8002276. Second author's research supported by NSF Grant MCS-8003600 A02.

\section{AmHerst College}

AMHERST, MA 01002

AND

SUNY

STONY BROOK, NY 11794 



\section{PACIFIC JOURNAL OF MATHEMATICS EDITORS}

\author{
DONALD BABBITT (Managing Editor) \\ University of California \\ Los Angeles, CA 90024 \\ J. DugundJI \\ University of Southern California \\ Los Angeles, CA 90089-1113 \\ R. FINN \\ Stanford University \\ Stanford, CA 94305 \\ HERMANN FLASChKa \\ University of Arizona \\ Tucson, AZ 85721
}

C. C. MOORE

University of California

Berkeley, CA 94720

Arthur Ogus

University of California

Berkeley, CA 94720

Hugo RossI

University of Utah

Salt Lake City, UT 84112

H. SAMELSON

Stanford University

Stanford, CA 94305

ASSOCIATE EDITORS
R. ARENS
E. F. BECKENBACH
B. H. NeUmanN
F. WOLF
K. YosHIDA (1906-1982)

\section{SUPPORTING INSTITUTIONS}
UNIVERSITY OF ARIZONA
UNIVERSITY OF BRITISH COLUMBIA
CALIFORNIA INSTITUTE OF TECHNOLOGY
UNIVERSITY OF CALIFORNIA
MONTANA STATE UNIVERSITY
UNIVERSITY OF NEVADA, RENO
NEW MEXICO STATE UNIVERSITY
OREGON STATE UNIVERSITY

\author{
UNIVERSITY OF OREGON \\ UNIVERSITY OF SOUTHERN CALIFORNIA \\ STANFORD UNIVERSITY \\ UNIVERSITY OF HAWAII \\ UNIVERSITY OF TOKYO \\ UNIVERSITY OF UTAH \\ WASHINGTON STATE UNIVERSITY \\ UNIVERSITY OF WASHINGTON
}

The Supporting Institutions listed above contribute to the cost of publication of this Journal, but they are not owners or publishers and have no responsibility for its content or policies.

Mathematical papers intended for publication in the Pacific Journal of Mathematics should be in typed form or offset-reproduced (not dittoed), double spaced with large margins. Please do not use built up fractions in the text of the manuscript. However, you may use them in the displayed equations. Underline Greek letters in red, German in green, and script in blue. The first paragraph must be capable of being used separately as a synopsis of the entire paper. In particular it should contain no bibliographic references. Please propose a heading for the odd numbered pages of less than 35 characters. Manuscripts, in triplicate, may be sent to any one of the editors. Please classify according to the scheme of Math. Reviews, Index to Vol. 39. Supply name and address of author to whom proofs should be sent. All other communications should be addressed to the managing editor, or Elaine Barth, University of California, Los Angeles, California 90024.

There are page-charges associated with articles appearing in the Pacific Journal of Mathematics. These charges are expected to be paid by the author's University, Government Agency or Company. If the author or authors do not have access to such Institutional support these charges are waived. Single authors will receive 50 free reprints; joint authors will receive a total of 100 free reprints. Additional copies may be obtained at cost in multiples of 50 .

The Pacific Journal of Mathematics is issued monthly as of January 1966. Regular subscription rate: $\$ 190.00$ a year (5 Vols., 10 issues). Special rate: $\$ 66.00$ a year to individual members of supporting institutions.

Subscriptions, orders for numbers issued in the last three calendar years, and changes of address should be sent to Pacific Journal of Mathematics, P.O. Box 969, Carmel Valley, CA 93924, U.S.A. Old back numbers obtainable from Kraus Periodicals Co., Route 100, Millwood, NY 10546.

The Pacific Journal of Mathematics at P.O. Box 969, Carmel Valley, CA 93924 (ISSN 0030-8730) publishes 5 volumes per year. Application to mail at Second-class postage rates is pending at Carmel Valley, California, and additional mailing offices. Postmaster: Send address changes to Pacific Journal of Mathematics, P.O. Box 969, Carmel Valley, CA 93924.

PUBLISHED BY PACIFIC JOURNAL OF MATHEMATICS, A NON-PROFIT CORPORATION

Copyright $\odot 1984$ by Pacific Journal of Mathematics 


\section{Pacific Journal of Mathematics}

Vol. 114, No. $2 \quad$ June, 1984

William Allen Adkins, A Harnack estimate for real normal surface

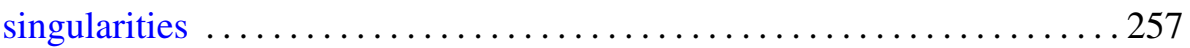

George E. Andrews, Multiple series Rogers-Ramanujan type identities . . . . 267

Didier Arnal, $*$ products and representations of nilpotent groups . . . . . . 285

David Cox and Walter Raymond Parry, Representations associated with

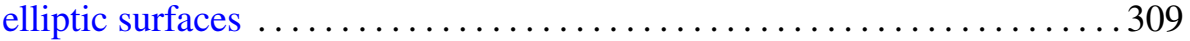

Joanne Marie Dombrowski, Tridiagonal matrix representations of cyclic

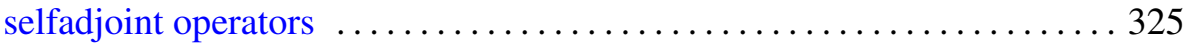

Ronald Dotzel, An Artin relation $(\bmod 2)$ for finite group actions on

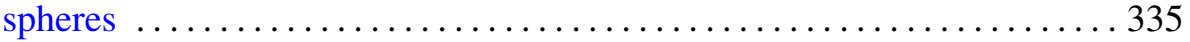

Leo Egghe, Convergence of adapted sequences of Pettis-integrable

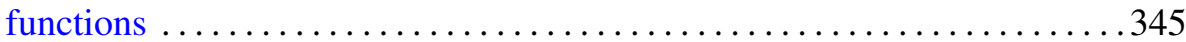

Rebecca A. Herb, Characters of induced representations and weighted

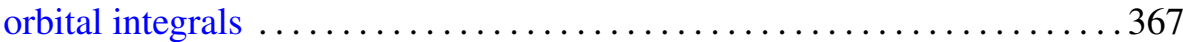

Steven M. Kahn, Cobordism obstructions to fibering manifolds over

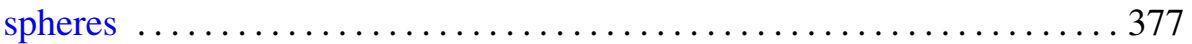

Robert D. Little, Projective space as a branched covering of the sphere with orientable branch set

Claude Schochet, Topological methods for $C^{*}$-algebras. III. Axiomatic

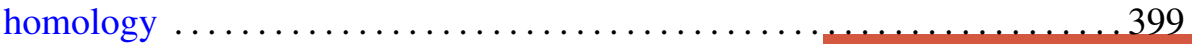

Claude Schochet, Topological methods for $C^{*}$-algebras. IV. $\bmod p$ homology

James M. Stormes, On the $K O$-orientability of complex projective varieties

Josephine Anne Ward, Characterization of homogeneous spaces and their norms 